Received: $\quad 2019.09 .08$ Accepted: 2019.10.31 Published: 2020.01.15

\section{Intussusception in an Immunocompromised Patient: A Case Report and Review of the Literature}

e-ISSN 1941-5923

DOI: 10.12659/AJCR.919974
Authors' Contribution:

Study Design A

Data Collection B

Statistical Analysis C

Data Interpretation D

Manuscript Preparation E

Literature Search F

Funds Collection G

\author{
ABCDEF Charles-Henri Wassmer \\ AE Ziad Abbassi \\ E Frédéric Ris \\ DE Thierry Berney
}

Department of Visceral and Transplant Surgery, University Hospital of Geneva, Geneva, Switzerland
Corresponding Author: Conflict of interest:
Charles-Henri Wassmer, e-mail: Charles-Henri.Wassmer@hcuge.ch

None declared

\footnotetext{
Patient: Female, 55-year-old

Final Diagnosis:

Symptoms:

Medication:

Clinical Procedure:

Specialty:

Objective:

Background:

Case Report:

Conclusions:

\author{
Septic shock with multi-organ failure \\ Respiratory distress \\ None \\ Laparotomy \\ Visceral Surgery
}

\section{Rare disease}

Intussusception in adults (Al) accounts for $1 \%$ of all cases of bowel obstruction. While pediatric intussusception is well known and almost always idiopathic, an underlying cause is usually found in adults. Indication for surgical treatment and intussusception reduction before resection remain controversial in Al. Here, we present an uncommon case of an immunocompromised patient who had multiple intussusceptions.

A 59-year-old woman, who had received a kidney-pancreas transplant for type 1 diabetes with end-stage renal failure, was admitted to our Intensive Care Unit for septic shock of suspected pulmonary origin. A thoracoabdominal CT scan demonstrated signs of bilateral pneumonia and multiple abdominal intussusceptions, for which she underwent surgery. Four intestinal intussusceptions were found. Manual desinvagination was performed without bowel resection. After surgery, the patient presented a new bowel obstruction, requiring a second surgery, showing recurrence of 1 intussusception. Segmental resection was indicated, but not performed because of the septic shock, requiring high-dose noradrenalin. The patient progressed toward multi-organ failure, leading to her death a few days later. An autopsy revealed that multiple adenomas were responsible for the intussusceptions.

This case confirms that Al is rarely a spontaneous disease and that the therapeutic strategy should be planned accordingly. There is currently no systematic approach for Al, and guidelines are needed to improve its management.
}

MeSH Keywords:

Full-text PDF:

Adult • Immunosuppression • Intussusception

https://www.amjcaserep.com/abstract/index/idArt/919974 


\section{Background}

Adult intussusception (Al) is a rare condition that accounts for $1 \%$ to $5 \%$ of all cases of bowel obstruction [1-7]. It is defined by the protrusion of a proximal bowel segment with his mesentery (intussusceptum) through the lumen of a distal bowel segment (intussuscipiens), resulting in occlusion [2,6-9]. Prolonged invagination causes edema, which can compromise the blood flow, resulting in ischemia and, if left untreated, necrosis and perforation $[2,10]$.

While pediatric intussusception is well known and mostly idiopathic, an underlying cause is found in $90 \%$ of adult cases $[1-4,6,7,11,12]$. Among the underlying lesions, malignancy accounts for $40 \%$ to $60 \%$ and predominates in the large bowel [4]. Furthermore, the risk of developing malignancies is increased in patients on immunosuppressive therapy [13].

Although clinical presentation in children is sudden, with acute symptoms such as abdominal pain, abdominal mass, and bloody mucoid stools (the classical triad), adult presentation can be insidious, with intermittent or even chronic symptoms, which can delay the diagnosis. Symptoms of intestinal obstruction are the most common. Others symptoms such as palpable mass or melena are less common $[1,3,4,9,12,14]$.

The management of $\mathrm{Al}$ is controversial regarding indications for surgery and the necessity or feasibility to reduce the intussusception before a resection. Here, by reporting an uncommon case of an immunocompromised patient who had 4 intussusceptions, and by performing a literature review, we attempt to clarify the management of this disease [15].

\section{Case Report}

A 59-year-old woman was transferred from a peripheral hospital to our Intensive Care Unit for suspected pulmonary septic shock. She had a history of type-1 diabetes with end-stage renal failure, for which she had received a combined kidneypancreas transplant 20 years ago and a second kidney transplant 7 years ago. She was on a mycophenolate mofetil and tacrolimus immunosuppressive regimen.

At the time of admission, she had received a 10-day course of intravenous antibiotics, with no clinical improvement. Both kidney and pancreas graft function were optimal, with normal creatinine level and glycemia within normal range. A thoracoabdominal CT scan demonstrated bilateral pleural effusion with signs of bilateral pneumonia and multiple abdominal intussusceptions (Figure 1). Immunosuppressive treatment was discontinued and it was decided to perform an exploratory laparotomy. Free intra-abdominal liquid and 4 sites of intussusception were found at 20, 30, 70 and 150 centimeters up from the ileocecal valve (Figure 2). All 4 intussusception sites presented an intramural lesion. Post-transplant lymphoproliferative disorder was suspected, and a biopsy of one of the lesions was taken. In the absence of bowel ischemia and to avoid extensive bowel resection, only manual desinvagination was performed. Histopathological analysis later revealed a low-grade tubulovillous adenoma.

Twelve days later, the patient presented a new episode of bowel obstruction, with a CT scan showing 1 intussusception. It also demonstrated progression of the pulmonary infection. The patient was taken again to the operating room, and a recurrence of the intussusception at the proximal site was found. Segmental resection was theoretically indicated, but, due to hemodynamic instability secondary to septic shock requiring vasoactive support, it was decided to refrain from respective surgery and to proceed again with a manual reduction of the invagination. Following surgery, the patient showed no clinical improvement. She progressed toward multiple organ failure (MOF) and eventually died 6 days after second surgery. Autopsy revealed multiple tubulovillous adenomas. It also showed massive necrosis of the pancreas graft, with thrombosis of the pancreatic artery. Finally, a pulmonary adenocarcinoma was found in the left lower lobe, with multiple bilateral pulmonary metastases and lymphangitic carcinomatosis.

\section{Discussion}

\section{Etiology}

$\mathrm{Al}$ is an unusual cause of intestinal obstruction. The mean age is 50 years, without a sex predominance [2]. It is the result of an organic cause in up to $90 \%$ of cases and idiopathic in about $10 \%[1,4,16]$. It can appear on every part of the gastrointestinal tract, but seems to occur more frequently at the junction between freely moving and retroperitoneally fixed segments or segments fixed by adhesions $[2,9,16]$. Idiopathic intussusceptions are mostly found in those regions, especially at the ileocecal junction [4,14].

Although some studies showed an equal distribution between small and large bowel, a predominance of small bowel intussusception is found (60\% to $81 \%)[1,4,8,12]$.

A malignant origin is found in about $1 / 3$ of small-bowel intussusceptions and 2/3 of large-bowel intussusceptions [17, 18]. Malignant lesions in the small bowel are usually secondary lesions, while in the large bowel, they are almost always primaries [2]. 


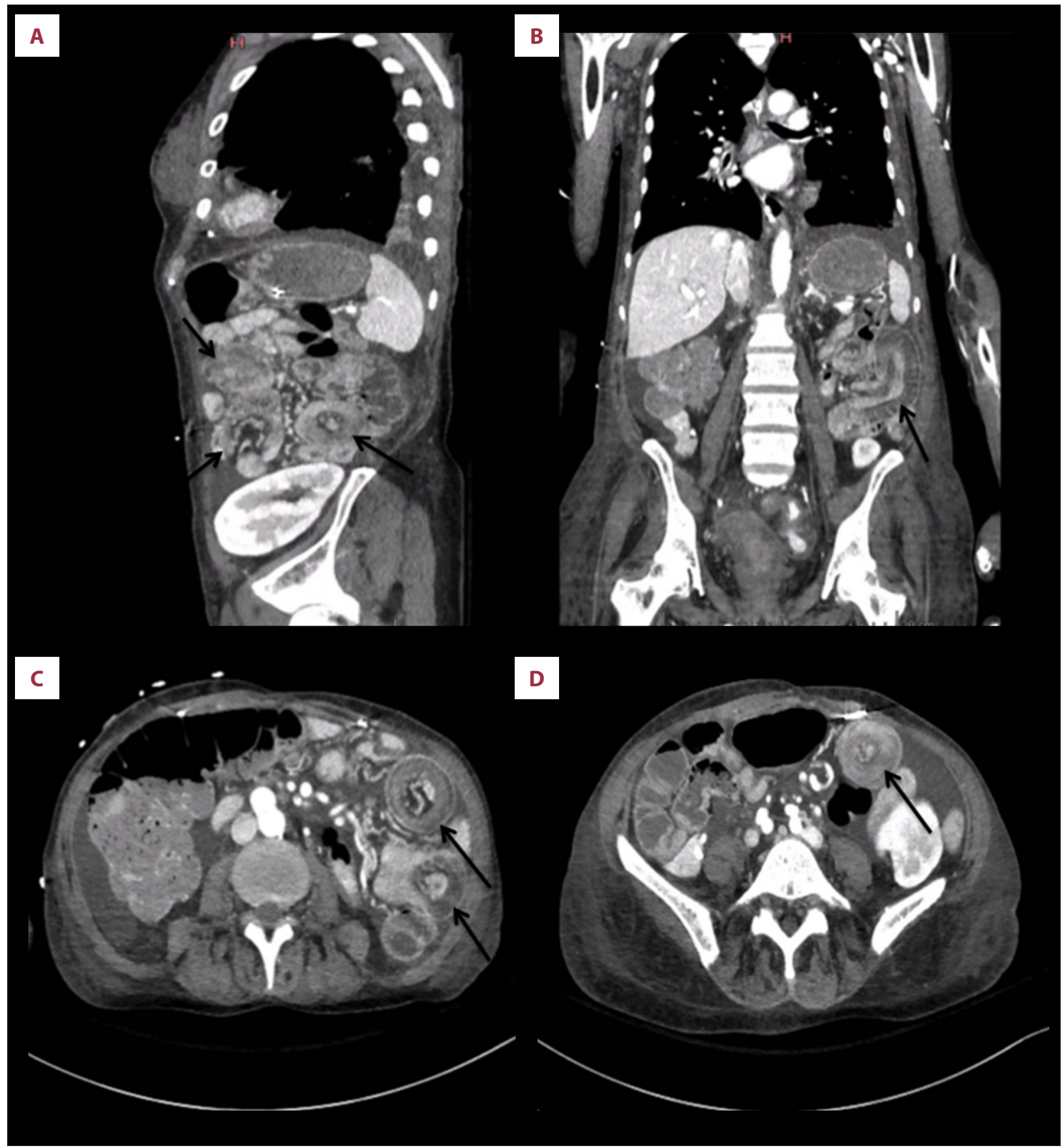

Figure 1. Sagittal (A), coronal (B), and transverse (C,D) views of abdominal CT showing the 4 intestinal intussusceptions, marked by black arrows. The classical "doughnut sign" can be seen on the sagittal and transverse views.

Many benign or malignant lesions can cause Al. Etiologies are summarized in Figure 3.

\section{Clinical presentation and investigation}

In contrast to children, clinical presentation in adults is generally nonspecific and insidious. Patients usually complain of abdominal pain, constipation, and vomiting, and, less frequently, melena or abdominal mass $[7,18]$. In case of malignancy, symptoms like weight loss, asthenia, and hematochezia can also be present [9]. Acute abdominal pain can occur, mostly in case of peritonitis resulting from necrosis and perforation. Finally, intussusception can be asymptomatic and is more frequently discovered with the increasing use of abdominal CT scans. 


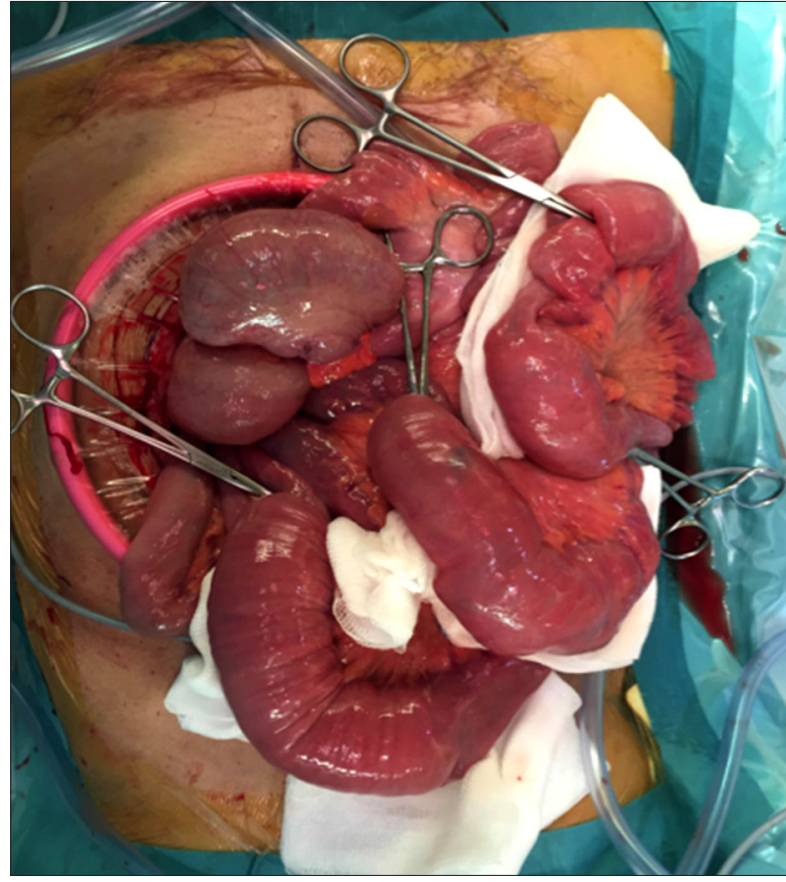

Figure 2. Intraoperative photo of the 4 intestinal intussusceptions marked with 4 forceps.

Diagnosis is rarely made based on patient history and clinical findings. Abdominal ultrasound is a useful tool to diagnose intussusception, especially in children. The characteristic findings include the "target" and "doughnut" signs on the transverse view and the "pseudo-kidney" sign on the lateral view $[4,7,18]$. Abdominal ultrasound is relatively inexpensive and widely available but operator-dependent, and the presence of air in the distended bowels can lead to difficult interpretation and poor image quality.

CT scan is the most accurate study, with a positive result in $70 \%$ to $100 \%$ of cases. The classic features are the "target" or "sausage-shaped" sign $[1,3,4,7,16,18]$. CT scans can identify the precise localization of the intussusception, as well as showing potential intraluminal masses or positive lymph nodes. Free fluid or air and signs of ischemia such as wall thickening and lack of enhancement of the bowel wall can also be easily seen. Radiological studies reported that an intussusception length $<4 \mathrm{~cm}$ and a diameter $<3.2 \mathrm{~cm}$ are more likely to resolve with conservative treatment $[20,21]$. On the contrary, 6 factors were found to be associated with an underlying cause: male sex, colonic involvement, visualization of a pathological lead point, abdominal pain, distal bowel diameter $\geq 27 \mathrm{~mm}$, and a wall thickness $\geq 3 \mathrm{~mm}$ [22].

\section{Management}

Many factors influence the management of Al: patient characteristics, age, small versus large bowel, emergency situations versus chronic presentation, and incidental findings.

Although treatment by contrast or air insufflation is the treatment of choice in the pediatric population, it is not indicated in adults [6]. Surgery is considered as the primary treatment

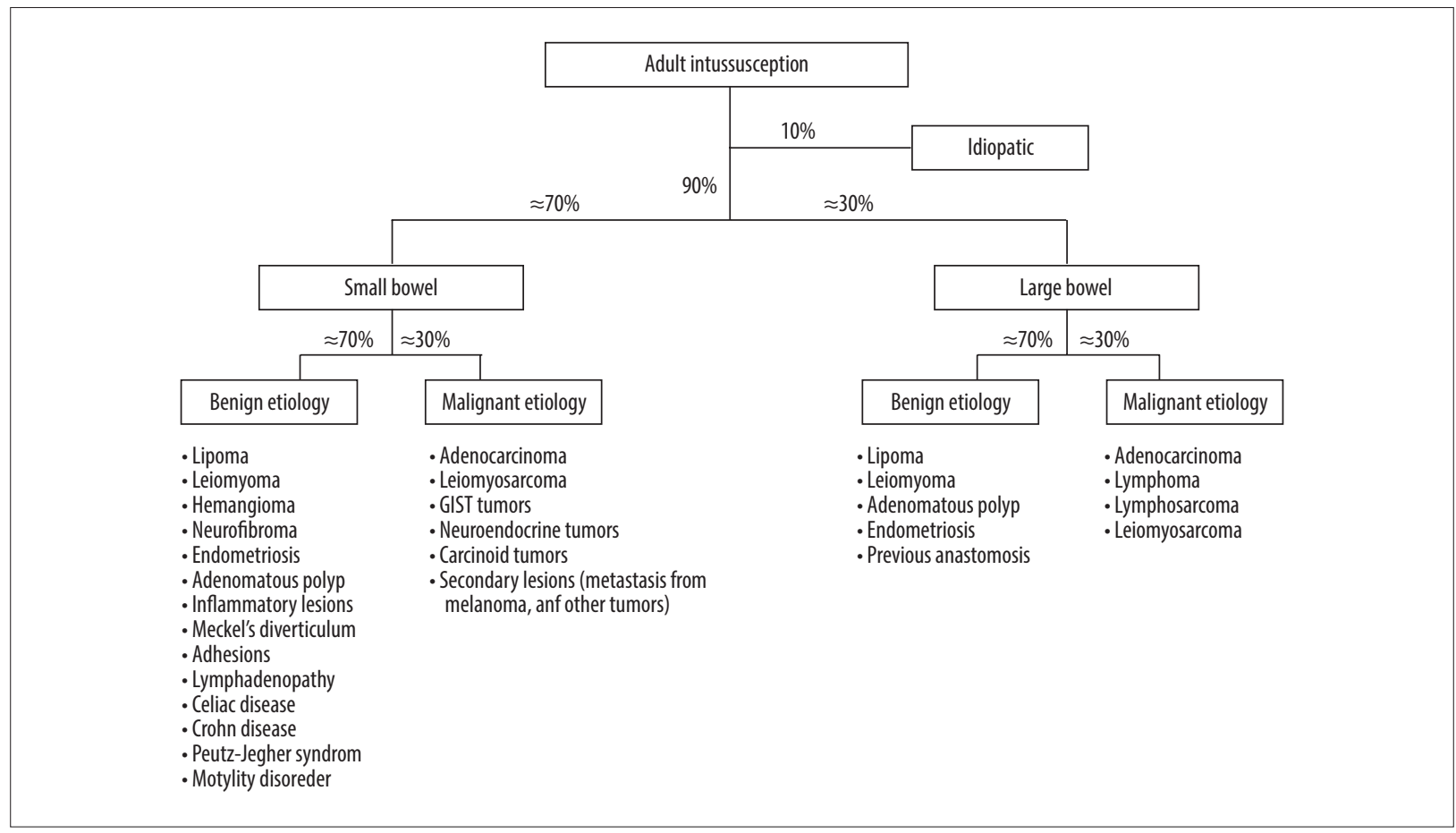

Figure 3. Adult intussusception etiologies. 


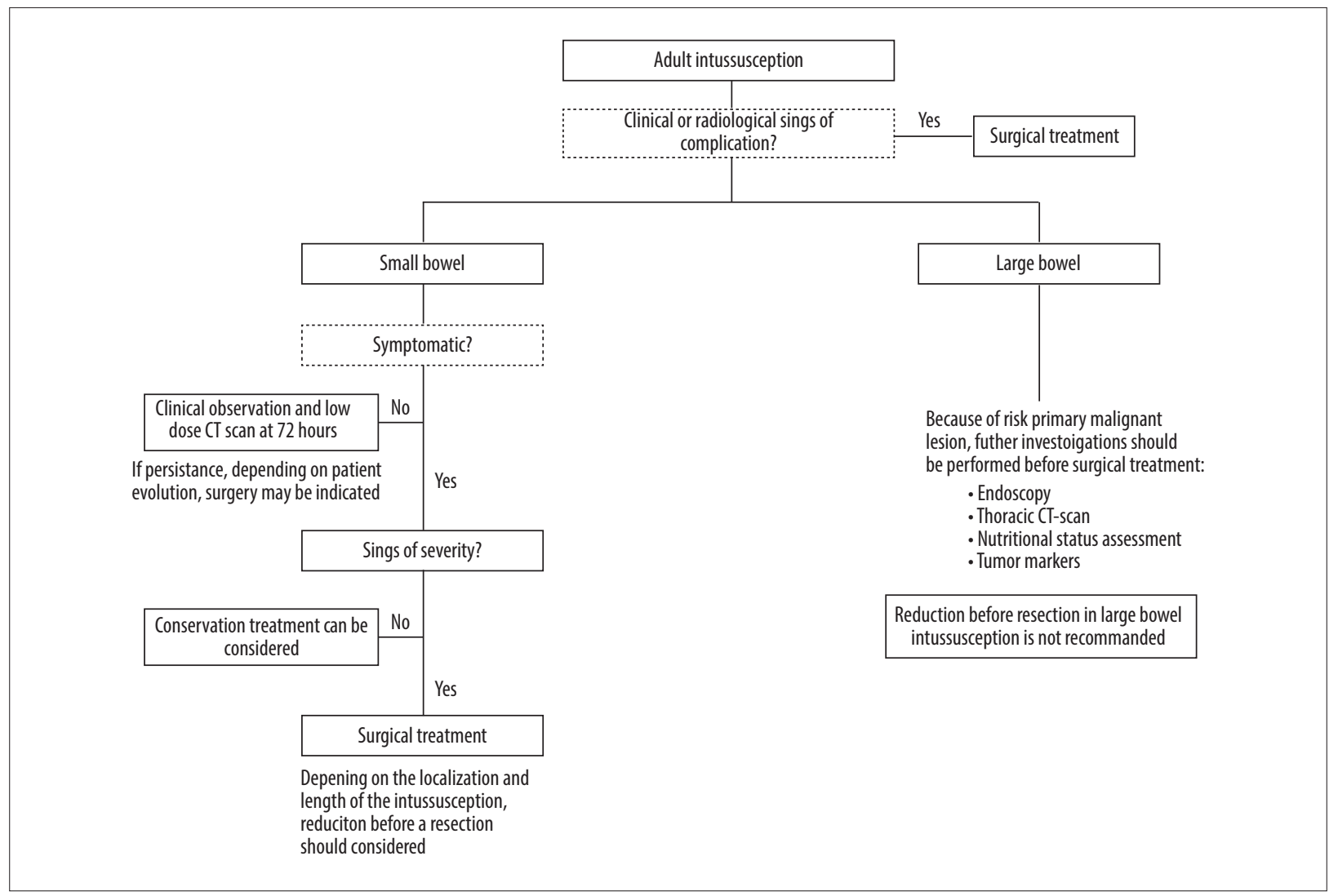

Figure 4. Proposed decision tree for management of adult patients with intussusception in small and large bowel.

of Al in symptomatic patients. The surgical approach can be open or minimally invasive, depending on emergency situations, surgeon experience or preference, and localization [6].

In case of large-bowel intussusception, a surgical approach should be chosen because of the risk of malignancy $[7,23]$. Depending on the perioperative findings, oncological resection may be performed, and perioperative histology assessment can help in the decision [6]. The intussusception should not be reduced because of the risks of vascular and intra-abdominal tumor cells dissemination and increased risks of perforation or anastomotic complications due to the weakened bowel wall $[2,4,6]$.

The management of symptomatic small bowel intussusception in adults is more controversial. Patient history, clinical presentation, and radiological findings are essential to make the most appropriate therapeutic decision. In the absence of any sign of complications or in case of an incidental finding, conservative treatment is recommended. A radiological examination must be carried out within 5 to 7 days to ensure resolution of the intussusception. In this case, a low-dose CT scan is the best choice [22].
If surgery is indicated, patients without any previous surgery should be treated by manual reduction before resection, to minimize the length of the resected segment. In case of multiple intussusceptions, a perioperative biopsy should be performed to identify the etiology and to avoid extensive bowel resection, which can result in a short-bowel syndrome. For example, multiple polyps can be seen in Peutz-Jeghers syndrome and can be responsible for repeated intussusceptions; a combination of polypectomy and limited bowel resection is the best approach in this situation [6].

In patients with a history of abdominal surgery, a "watch and wait approach" is acceptable since adhesions can cause the intussusception. If symptoms do not resolve spontaneously, surgery is the treatment of choice.

In all cases, if clinical history and/or radiological findings show signs of complications (e.g., acute abdominal pain, weight loss, asthenia, free intra-abdominal air or fluid, and lack of parietal enhancement), surgery has to be considered. If an oncological etiology is suspected, and if clinical presentation allows it, a complete oncological assessment should be carried out. To help physicians in patient management, we developed a decision tree (Figure 4). 
Patients receiving immunosuppressive treatment are at higher risk of developing malignancies. The most common cancers seen in immunosuppressed transplant recipients are non-melanoma skin cancer and non-Hodgkin lymphoma. Several other types of malignancies have higher relative risks. Lung cancer, as seen in the present case, also has a significantly higher incidence in immunosuppressed patients, with a relative risk of 1.4 [24]. Tubulovillous adenomas have not been associated with immunosuppressive treatment, but have the potential to progress to adenocarcinoma in the colon [25].

In the present case, we faced multiple intussusceptions in an immunocompromised patient. In the absence of ischemia, because the patient was in severe sepsis with a high risk of anastomotic leakage, and in view of the length of intestine involved, bowel resection was avoided. The histological assessment performed perioperatively showed no sign of malignancy but did show several adenomas. The patient had a recurrence, but because of her general condition, resection and anastomosis would have been too risky. The most likely cause of death was the pulmonary insufficiency on bilateral pneumonia and the advanced lung cancer. The autopsy also showed necrosis of the pancreatic graft with thrombosis of the artery. Despite this discovery, the patient had preserved pancreatic graft function, and, notably, no exogenous insulin treatment was required. This was most probably the result of the hypercoagulable state secondary to septic shock.
It is unlikely that the intussusceptions were the reason for MOF; therefore, it is unlikely that a more aggressive approach during the first surgery would have improved the outcome. On the contrary, the poor hemodynamic status of the patient combined with multiple bowel resections/anastomosis would have put the patient at a high risk of complications.

\section{Conclusions}

Intussusception is a rare condition in adults, with various clinical presentations. Abdominal CT scan is the imaging modality of choice. There is currently no clinical or scientific evidence indicating a preferred treatment. Therefore, each case should be assessed individually. The presented case confirms that adult intussusception is rarely a spontaneous disease and that the therapeutic strategy should be planned accordingly.

\section{Acknowledgements}

The authors thank the staff of the Intensive Care Unit of Geneva University Hospital for their help with this case.

\section{Department and Institution where work was done}

\section{Geneva University Hospital, Geneva, Switzerland}

\section{Conflict of interest}

None.

12. Hanan B, Diniz TR, da Luz MM et al: Intussusception in adults: A retrospective study. Colorectal Dis, 2010; 12(6): 574-78

13. Grulich AE, van Leeuwen MT, Falster MO, Vajdic CM: Incidence of cancers in people with HIV/AIDS compared with immunosuppressed transplant recipients: A meta-analysis. Lancet, 2007; 370(9581): 59-67

14. Honjo $\mathrm{H}$, Mike $\mathrm{M}$, Kusanagi $\mathrm{H}$, Kano N: Adult intussusception: A retrospective review. World J Surg, 2015; 39(1): 134-38

15. Wassmer CH, Abbassi Z, Ris F, Berney T: Intussusception in an immuno-compromised patient: Case report and review of the literature. ESSR Abstracts. Eur Surg Res, 2019; 60(Suppl. 2): 214

16. Maghrebi H, Makni A, Rhaiem R et al: Adult intussusceptions: Clinical presentation, diagnosis and therapeutic management. Int J Surg Case Rep, 2017; 33: 163-66

17. Barussaud $M$, Regenet N, Briennon X et al: Clinical spectrum and surgical approach of adult intussusceptions: A multicentric study. Int J Colorectal Dis, 2006; 21(8): 834-39

18. Renzulli P, Candinas D: Idiopathic small-bowel intussusception in an adult CMAJ, 2010; 182(3): E148

19. Zubaidi A, Al-Saif F, Silverman R: Adult intussusception: A retrospective review. Dis Colon Rectum, 2006; 49(10): 1546-51

20. Lvoff N, Breiman RS, Coakley FV et al: Distinguishing features of self-limiting adult small-bowel intussusception identified at CT. Radiology, 2003; 227(1): 68-72

21. Rea JD, Lockhart ME, Yarbrough DE et al: Approach to management of intussusception in adults: A new paradigm in the computed tomography era. Am Surg, 2007; 73(11): 1098-105 
22. Tan HL, Koh YX, Taufik M et al: A clinical scoring system to predict the clinical sequelae of computed tomography diagnosed intussusception. World J Surg, 2018; 42(3): 682-87

23. Goh BK, Quah HM, Chow PK et al: Predictive factors of malignancy in adults with intussusception. World J Surg, 2006; 30(7): 1300-4
24. Collett D, Mumford L, Banner NR et al: Comparison of the incidence of malignancy in recipients of different types of organ: A UK Registry audit. Am J Transplant, 2010; 10(8): 1889-96

25. Kalimuthu SN, Chelliah A, Chetty R: From traditional serrated adenoma to tubulovillous adenoma and beyond. World J Gastrointest Oncol, 2016; 8(12): 805-9 